\title{
Immunogenomic pathways associated with cytotoxic lymphocyte infiltration and survival in colorectal cancer
}

\author{
Yuanyuan Shen², Yue Guan², Justin J. Hummel ${ }^{2}$, Chi-Ren Shyu ${ }^{2}$ and Jonathan B. Mitchem ${ }^{1,2,3^{*}}$ (D)
}

\begin{abstract}
Background: Colorectal cancer (CRC) is the second leading cancer killer in the US today and patients with metastatic disease have only a 14\% 5-year survival. One of the most impactful recent advances in cancer therapy, immune checkpoint inhibition, has not been shown to be effective for the majority of these patients. In this study, we use The Cancer Genome Atlas (TCGA) and recently developed informatic-based tools to identify targets for immune based therapy in colorectal cancer patients.

Methods: Open access, pre-processed (level 3) mRNA data and clinical data from colorectal patients from the TCGA was downloaded from FireCloud. Using the Microenvironment Cell Populations-Counter method (MCP-Counter), cytotoxic lymphocyte scores were calculated for all patients. Patients were then grouped by cytotoxic lymphocyte score (High vs Low), pathologic stage, and location to identify differentially expressed genes. Pathway enrichment analysis was performed using Reactome to determine differentially expressed genes associated with immune pathways. Survival analysis was performed with identified differentially expressed genes.
\end{abstract}

Results: In the TCGA dataset, there are 461 colon and 172 rectal cancer patients. After stratifying patients by cytotoxic lymphocyte score, anatomical location, and stage, we found a significant number of differentially expressed genes. We identified one pathway, "immunoregulatory interactions between a lymphoid and nonlymphoid cell", that was highly enriched and included in all tumor locations and stages. Survival analysis performed with differentially expressed genes in this pathway identified 21 different genes associated with survival and cytotoxic lymphocyte infiltration, with $\sim 70 \%$ of these genes occurring in the metastatic rightsided CRC group. Specifically, all genes associated with survival in the metastatic right-sided colorectal cancer group with low cytotoxic lymphocyte scores positively impacted survival.

Conclusions: Utilizing the TCGA, a publicly available dataset, and informatics-based analyses, we identified potential targets to improve immune based therapy in colorectal cancer. Additionally, we note the most targets in metastatic right-sided CRC patients, the patient group with the worst predicted survival. The results from this study demonstrate the ability of informatics-based analytic techniques to identify new therapeutic targets as well as improve patient selection for intervention, helping us to achieve the goals of precisionbased oncology.

Keywords: Colorectal cancer, Colon, Rectum, Immunotherapy, Immunogenomics, Immuno-oncology, Cytotoxic lymphocytes

\footnotetext{
* Correspondence: mitchemj@health.missouri.edu

${ }^{1}$ Harry S. Truman Memorial Veterans' Hospital, Columbia, MO, USA

${ }^{2}$ Institute for Data Science and Informatics, University of Missouri, 238 Naka

Hall, Columbia, MO 65211-2060, USA

Full list of author information is available at the end of the article
}

(c) The Author(s). 2020 Open Access This article is distributed under the terms of the Creative Commons Attribution 4.0 International License (http://creativecommons.org/licenses/by/4.0/), which permits unrestricted use, distribution, and reproduction in any medium, provided you give appropriate credit to the original author(s) and the source, provide a link to the Creative Commons license, and indicate if changes were made. The Creative Commons Public Domain Dedication waiver (http://creativecommons.org/publicdomain/zero/1.0/) applies to the data made available in this article, unless otherwise stated. 


\section{Background}

Despite recent advances in detection and therapy, colorectal cancer (CRC) remains the second leading cause of cancer-related death in the US [1]. Immune based therapies such as immune checkpoint inhibition have recently made significant advances in a number of difficult to treat malignancies like non-small cell lung cancer, melanoma, and renal cell cancer [2]. However, these results have not yet extended to the majority of patients with CRC [3]. This is despite significant data that antitumor immunity is important for prognosis and treatment response in these patients $[4,5]$. This suggests that there is significant progress to be made in the application of immune based therapy in CRC.

When considering immune based treatments, a critical factor is effective tumor infiltration of cytotoxic lymphocytes [6]. In colorectal cancer, this is evident as patients who demonstrate response to immune checkpoint inhibition and currently have an FDA approved indication for this therapy, are those with microsatellite instability-high (MSI-H) tumors [7]. These tumors are characterized by high mutational load, neoepitope formation, and an intense lymphocytic infiltrate when compared to microsatellite stable (MSS) tumors [8]. Microsatellite instabilityhigh tumors, however, are also associated with increased mutations in immune related genes and expression of negative regulatory genes, demonstrating that tumors try to dampen the immune response by multiple pathways $[9,10]$. Additionally, recent studies have suggested that the use of other markers including lymphocyte infiltration and tumor mutational burden may better predict survival and the potential for response to immune based therapy $[5,11]$. It is therefore critical to develop a better understanding of immune resistance mechanisms to improve therapy in colorectal cancer patients.

In the current era of precision medicine, research is concentrated on providing more effective treatments by focusing on patient specific factors. This is particularly important in colorectal cancer, as subsets of patients responsive to targeted therapy, immune-based therapy, and chemotherapy have previously been identified $[3,12$, 13]. Colorectal cancer, however, is a heterogeneous disease made up of multiple subgroups [14]. Even simple clinical characteristics often overlooked in molecular studies, such as anatomic location, are important for prognosis $[15,16]$. Despite these differences in subtype and clinical characteristics, $\mathrm{T}$ lymphocyte infiltration has been demonstrated to be important for prognosis [5].

Data repositories such as The Cancer Genome Atlas (TCGA) allow for the in-depth study of patients on a molecular and clinical basis [17]. Recently, a novel computational method for predicting the abundance of different cells within the tumor microenvironment using RNA-seq data was developed and validated with histologic specimens called the Microenvironment Cell PopulationCounter (MCP-Counter, [4]). This method allows for an effective comparison of the composition and pathways associated with cellular infiltration in the tumor microenvironment, improving over other methods primarily based on microarray data and gene set enrichment analysis. In this study, we use the MCP-counter program to create tumor cytotoxic lymphocyte (CL) abundance scores. After grouping patients based on cytotoxic lymphocyte abundance score, stage, and tumor location, we found one immune pathway that was highly enriched at all tumor locations and stages, the "Immunoregulatory interactions between a Lymphoid and a non-Lymphoid cell" pathway, suggesting specific targets to improve immune based therapy in colorectal cancer patients.

\section{Methods}

The cancer genome atlas data access and processing

The data shown here is based upon data originally generated and organized by FireCloud from the Broad Institute (Fig. 1). Full permission access transcriptomic data was obtained from dbGAP. We downloaded CRC patients' open access, pre-processed mRNA expression data (level 3 data, [18]) from both platforms, IlluminaHiSeq and Illumina-GA; as well as mRNA RNA-Seq by Expectation Maximization (RSEM) normalized data; and patients' clinical data from the cohorts TCGA_COAD_ ControlledAccess and TCGA_READ_ControlledAccess by gsutil Tool.

We integrated pertinent clinical data (age, gender, microsatellite status, anatomical location, pathologic stage, Tumor Node and Metastasis (TNM) classification, days to last follow up, and vital status), and RNA-seq by participant ID. Each CRC patient has pre-identified microsatellite status labeled as "microsatellite instability test results". Thirty-four patients with colon cancer and 18 patients with rectal cancer were excluded due to missing information including indeterminate microsatellite status, unclear anatomical location or pathologic stage, and unmatched RNA-seq data. Then for every patient, we implemented the $\mathrm{R}$ package Microenvironment Cell Populations-Counter on the RNA-Seq by Expectation Maximization (RSEM) normalized RNA-seq data to create cell type abundance scores [4]. There are 10 cell populations simultaneously quantified in the tumor microenvironment, including 8 immune cell populations ( $\mathrm{T}$ cells, CD8 T cells, Cytotoxic lymphocytes, NK cells, B lineage, Monocytic lineage, Myeloid dendritic cells, Neutrophils), endothelial cells and fibroblasts. Specifically, the gene set for cytotoxic lymphocytes includes the genes: CD8A, EOMES, FGFBP2, GNLY, KLRC3, KLRC4, and KLRD1. We used the median value of the cytotoxic lymphocytes (CL) score to group the patients into high ( $\geq$ median value, CL-High) and low ( $<$ median value, CL-Low) groups. We then grouped patients by anatomical location and stratified by cytotoxic lymphocyte 


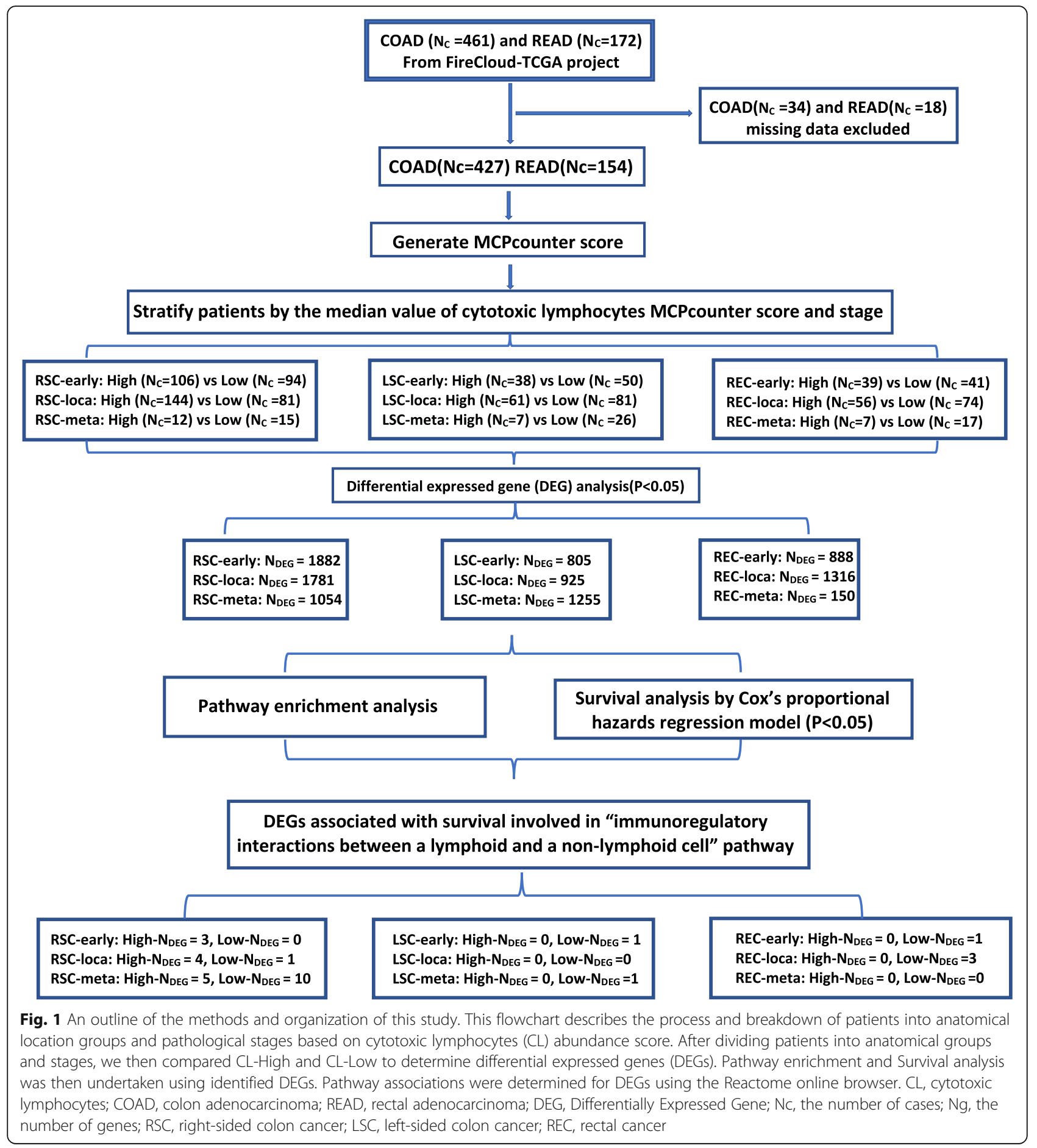

score and pathologic stage. For consistency in clinical intervention while increasing group size, we assigned pathologic stage I-II to "early" stage, stage I-III as "localized", and stage IV as "metastatic" (Fig. 1). Patients were grouped by tumor subsite: tumors located in the cecum, ascending colon, hepatic flexure, and transverse colon were categorized as having right-sided colon cancer (Fig. 1, RSC); tumors located in the splenic flexure, descending colon, sigmoid colon or rectosigmoid junction were categorized as having left-sided colon cancers (Fig. 1, LSC); patients with tumors located in the rectum were kept in this group (Fig. 1, REC).

Demographics and clinicopathologic characteristic analysis

Demographic, clinical, and pathologic characteristics were retrieved as stated above. Statistical analyses were 
performed using Prism 7 (GraphPad Software). Patients' basic clinical features were summarized by descriptive statistics, including means and standard deviation, and an unpaired t-test was used for normally distributed continuous data. Categorical variables were compared using Fisher's exact and chi-square tests. A $p$ value < 0.05 was considered statistically significant.

\section{RNA-seq differential gene expression analysis}

RNA-seq differential gene expression analysis was performed with the edgeR package using the raw data downloaded from the Illumina- HiSeq and Illumina-GA platforms [19]. Differentially expressed genes were defined as genes with an absolute fold change $>1$ between patients with high and low cytotoxic lymphocyte scores with a $p$ value $<0.05$ [19]. Genes with Benjamini-Hochberg adjusted False Discovery Rate $($ FDR $)<0.05$ were considered to be significantly differentially expressed for further steps. For each cohort, we identified 20, 531 total genes by RNA-seq raw counts. The Reactome online browser was used to identify immune functional differentially expressed genes [20]. Figure 1 outlines this process.

\section{Pathway enrichment and survival analysis}

Pathway enrichment analysis was performed to evaluate the pathways associated with differentially expressed genes. The genes included in the MCP-counter cytotoxic lymphocyte gene panel (CD8A, EOMES, FGFBP2, GNLY, KLRC3, KLRC4, and KLRD1) were excluded from pathway enrichment analysis. Dotplot was used to illustrate the comparison of enriched Reactome pathways among differentially expressed genes in each location and stage. These results were analyzed by clusterProfiler, DOSE, and ReactomePA R packages. Next, we performed survival analyses using the identified differentially expressed genes. Patients were organized by stage and location as outlined above. The normalized RNA-seq data of differentially expressed genes used for survival analysis was processed using the Survival $R$ package [20]. For each differentially expressed gene, if the normalized gene expression value was more than the median level, we labeled it as "high," and otherwise as "low." The Kaplan-Meier survival curves generated were assessed by the Cox regression model for each immune functional differentially expressed gene using the Survminer R package [21, 22]. The survival curves of patients with high gene expression and low gene expression were compared by log-rank test. For each patient, overall survival (OS) was used as the endpoint, either the days from diagnosis to death, or to the last follow-up (Fig. 1).

The Reactome pathway online browser was used to identify differentially expressed genes associated with survival in sub-pathways of "immunoregulatory interactions between a lymphoid and a non-lymphoid cell pathway" (Fig. 1).

\section{Results}

\section{Patient characteristics}

In the most recently updated TCGA dataset (June 01, 2016), there are 461 colon cancer (COAD) and 172 rectal cancer (READ) cases (Fig. 1, [17]). Thirty-four patients with colon cancer and 18 patients with rectal cancer were excluded due to missing information. The RNA-seq data with matched clinical data were integrated from COAD patients $(\mathrm{Nc}=427)$ and READ patients $(\mathrm{Nc}=154)$. Cytotoxic lymphocyte abundance scores were generated using the MCP-counter method [4]. Patients were then separated based on the median cytotoxic lymphocyte score (26.72; 95\% CI: 24.1-30.1, Additional file 1: Table S1, values and analysis included in Additional file 2). Patients with cytotoxic lymphocyte scores $\geq$ the median were classified as cytotoxic lymphocyte-high (CL-High) and those with scores $<$ the median were classified as cytotoxic lymphocyte-low (CL-Low). We then confirmed that there was a significant difference in cytotoxic lymphocyte scores between CL-High and CL-Low groups $(73.66 \pm 64.2$ v $14.07 \pm 6.69, p<0.0001$, Additional file 1: Figure S1). Colorectal cancer patients were then separated by anatomical location, cytotoxic lymphocyte score, and stage (Fig. 1). The demographic, clinical, and pathologic characteristics of each patient cohort is summarized in Table 1. Microsatellite status composition of patients with CL-High and CL-Low tumors was significantly different in the right-sided colon cancer $(p<$ $0.0001)$ and rectal cancer $(p=0.0264)$ groups with more MSI-H patients among the CL-High patients at both locations (Table 1). Additionally, cytotoxic lymphocyte scores correlated significantly with pathologic tumor stage in right-sided colon cancer $(p=0.0278)$ and rectal cancer $(p=0.0279)$ patients, but not in left-sided colon cancer patients (Table 1). This analysis demonstrates that there are significant differences based on tumor location, suggesting that this variable is an important consideration when analyzing patient data [15, 23].

\section{Differential gene expression analysis}

For each cohort, we next performed RNA-seq differential gene expression analysis. Expression of 20,531 genes was determined for each tumor sample from the TCGA. Then gene expression was compared between patients with CL-High and CL-Low tumors in each cohort based on tumor location and stage using the edgeR package (analysis included in Additional file 2). In right-sided early, localized, and metastatic colon cancer patients, 1882,1781 , and 1054 differentially expressed genes were observed, respectively. In left-sided patients, 805, 925, and 1255 genes were differentially expressed in each stage. And in rectal cancer patients, 888, 1316 and 150 genes were differentially expressed at each stage (Fig. 1). In the left-sided group, differentially expressed genes were highest in the metastatic cohort; however, in right- 
Table 1 Patient Characteristics

\begin{tabular}{|c|c|c|c|}
\hline Characteristic & High & Low & $P$ value \\
\hline \multicolumn{4}{|l|}{ Gender, n } \\
\hline RSC & 156 & 96 & 0.3637 \\
\hline Male & 77 & 54 & \\
\hline Female & 77 & 42 & \\
\hline LSC & 68 & 106 & 0.1231 \\
\hline Male & 41 & 51 & \\
\hline Female & 27 & 55 & \\
\hline REC & 63 & 91 & 0.5118 \\
\hline Male & 37 & 48 & \\
\hline Female & 26 & 43 & \\
\hline \multicolumn{4}{|l|}{ MS, n } \\
\hline RSC & 156 & 96 & $<0.0001^{*}$ \\
\hline MSS/MSI-L & 92 & 89 & \\
\hline MSI-H & 64 & 7 & \\
\hline LSC & 68 & 106 & 0.0771 \\
\hline MSS/MSI-L & 64 & 105 & \\
\hline MSI-H & 4 & 1 & \\
\hline REC & 63 & 91 & $0.0264^{*}$ \\
\hline MSS/MSI-L & 59 & 91 & \\
\hline MSI-H & 4 & 0 & \\
\hline \multicolumn{4}{|l|}{ Age,Mean \pm SD } \\
\hline RSC & $70 \pm 13.69$ & $67 \pm 12.95$ & 0.2222 \\
\hline LSC & $66 \pm 11.11$ & $64 \pm 13.07$ & 0.4267 \\
\hline REC & $64 \pm 10.56$ & $65 \pm 12.32$ & 0.81 \\
\hline \multicolumn{4}{|c|}{ Pathologic stage, n (\%) } \\
\hline RSC & 156 & 96 & $0.0278^{*}$ \\
\hline 1 & $33(21.2 \%)$ & $11(11.5 \%)$ & \\
\hline$\|$ & $73(46.8 \%)$ & 38(39.6\%) & \\
\hline III & $38(24.4 \%)$ & $32(33.3 \%)$ & \\
\hline IV & $12(7.7 \%)$ & 15(15.6\%) & \\
\hline LSC & 68 & 106 & 0.1374 \\
\hline I & $12(17.6 \%)$ & $17(16.0 \%)$ & \\
\hline$\|$ & $26(38.2 \%)$ & 33(31.1\%) & \\
\hline III & 23(33.8\%) & $30(28.3 \%)$ & \\
\hline IV & $7(10.3 \%)$ & $26(24.5 \%)$ & \\
\hline REC & 63 & 91 & \\
\hline 1 & 10(15.9\%) & $20(22.0 \%)$ & $0.0279^{*}$ \\
\hline$\|$ & $29(46.0 \%)$ & $21(23.1 \%)$ & \\
\hline III & $17(27.0 \%)$ & $33(36.3 \%)$ & \\
\hline IV & 7(11.1\%) & $17(18.7 \%)$ & \\
\hline
\end{tabular}

RSC Right-sided colon cancer, LSC Left-sided colon cancer, REC Rectal cancer, MSS Microsatellite stable, MSI-L Microsatellite instability-low, MSI-H Microsatellite instability-high

*Indicates statistically significant difference $(p<0.05)$ sided and rectal cancer patients, the metastatic cohort had the lowest number of differentially expressed genes. This again suggests that both tumor location and stage are important considerations when analyzing alterations in gene expression. Differentially expressed genes found in the above analysis were subsequently imported into the Reactome Pathway Browser to determine involvement in immune related functional pathways (Fig. 1, [15]). Interestingly, despite significant variation in the number of differentially expressed genes, the ratio of genes associated with immune function was similar in all sites and stages.

\section{Pathway enrichment and survival analysis}

To further determine whether there were overlapping pathways associated with cytotoxic lymphocyte infiltration in colorectal cancer, we then compared the Reactome pathway enrichment analysis at each location based on stage. Using the $p$ value adjusted for false discovery rate and the ratio of differentially expressed genes in each pathway, we found that the "immunoregulatory interactions between a lymphoid and a non-lymphoid cell" was the most highly enriched pathway in early and local patients at all tumor locations. Additionally, this was the most highly enriched pathway in patients with metastatic right-sided cancer. This pathway was also among the top pathways enriched among patients with metastatic left-sided colon cancer and rectal cancer (Fig. 2, data and analysis included in Additional file 2). This suggests that despite significant heterogeneity among subjects, redundant pathways of deregulation may be conserved across stage and location.

To further understand the potential for targetable genes within this pathway, we then took differentially expressed genes in the "immunoregulatory interactions between a lymphoid and non-lymphoid cell" pathway and performed a survival analysis using the KaplanMeier method and Cox-Proportional Hazards Model based on differentially expressed gene, location, cytotoxic lymphocyte score, and pathologic stage (Fig. 3, Table 2, remaining survival curves and analysis included in Additional file 2). There are a total of 297 genes included in this pathway, and we found 21 (7.1\%) unique genes associated with survival in this pathway. As Fig. 1 demonstrates, the number of differentially expressed genes were variable with most genes associated with survival in the right-sided colon cancer group. Additionally, the positive and negative impact of differentially expressed genes on survival depended on cytotoxic lymphocyte abundance scores. The majority of genes associated with a positive impact on survival (bold in Table 2) were in the CL-Low group whereas the majority of genes with a negative impact on survival (italicized in Table 2) were found in the CL-High group. In CL-High 


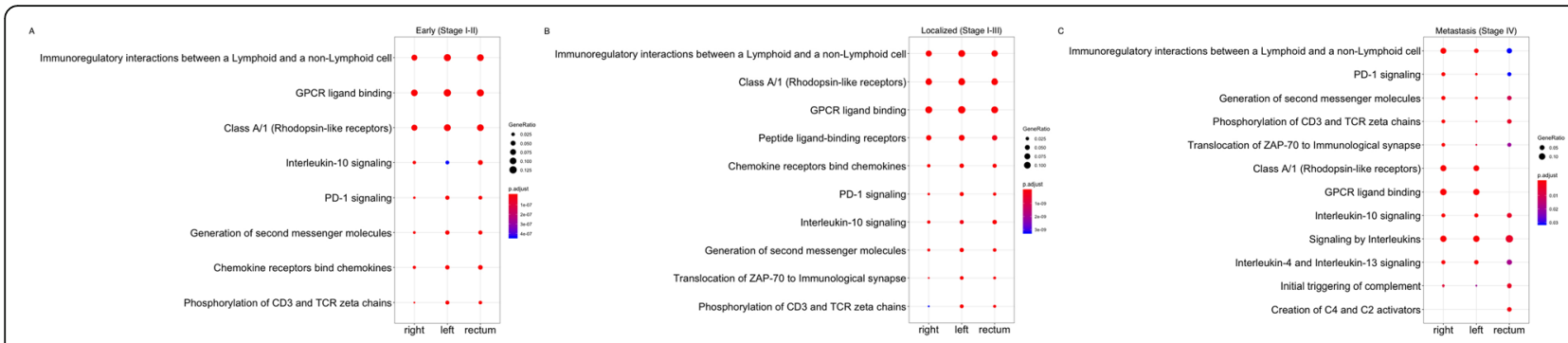

Fig. 2 Pathway Enrichment analysis based on tumor location and stage. Pathway enrichment was ranked using a composite of the adjusted $p$ value for false discovery rate (color) and gene ratio (size). a pathway enrichment for early stage CRC (Stage I and II) based on location; $\mathbf{b}$ pathway enrichment analysis for localized CRC (Stages I-III) based on location; c pathway enrichment analysis for metastatic stage CRC (Stage IV) based on location. This analysis showed that the "immunoregulatory interactions between a lymphoid and a non-lymphoid cell" was the most highly enriched pathway in early and local patients at all sites. Additionally, this was the most highly enriched pathway in patients with metastatic right-sided cancer. This pathway was also among the top pathways enriched among patients with metastatic left-sided colon cancer and rectal cancer. (Input data included in Additional file 2)

right-sided colon cancer patients with metastatic disease, all differentially expressed genes had a negative impact on survival; however, all immune functional differentially expressed genes in the CL-Low group had a positive impact on survival. Often, patients with rectal cancer and left-sided cancer are considered to have similar disease biologically. While we found few differentially expressed genes in this pathway associated with survival in the leftsided and rectal cancer groups, differentially expressed genes in the left-sided colon cancer group primarily had a positive impact on survival with the converse being true in patients in the rectal cancer group. Together this data demonstrates that even within conserved immune pathways, there is significant heterogeneity in the impact on patient survival. This further suggests the importance of a patient centered approach for the application of immune based therapy in colorectal cancer.

\section{Discussion}

Cytotoxic lymphocyte infiltration is critical for response to immune based therapy [6] and has been shown to predict survival and treatment response in colorectal cancer $[4,5]$. A better understanding of potential targets is critical for the improvement of immune based therapy in colorectal cancer as currently utilized therapy is not effective in the majority of patients. Therefore, in this study we have combined publicly available data resources with computational methods to focus on genes that may have an impact both on tumor associated cytotoxic lymphocytes and survival [4]. Comparing patients with high and low cytotoxic lymphocyte abundance scores, we found many differentially expressed genes at all tumor locations and stages. Unsurprisingly, the group with the highest number of immune related differentially expressed genes was the right-sided colon cancer group. This may be a reflection of the higher number of MSI-H patients in this group, which is expected to have a higher mutation rate, and therefore, potentially more genes with altered expression.

To further define potential therapeutic targets, we then performed pathway enrichment analysis. In this analysis, we found the pathway, "immunoregulatory interactions between a lymphoid and non-lymphoid cell", was among the most highly enriched and altered in all sites and stages. A few pathways were occasionally more highly enriched, however were not affected at all sites or stages, therefore, we chose to focus on this pathway. The "immunoregulatory interactions between a lymphoid and non-lymphoid cell" pathway involves a number of cell surface signaling pathways that are involved in the regulation of anti-tumor immunity [20]. After performing survival analyses using the differentially expressed genes from this pathway, we found the majority of genes affecting survival were in the right-sided patient group consistent with the differential gene expression analysis. Patients with right-sided colon cancer have significantly worse survival than other tumor locations at all stages, and right-sided colon cancer patients with metastatic disease demonstrate poorer survival with current chemotherapy regimens. This group, therefore, likely represents the group with the most important need for new therapeutic options [15, 23]. There was, however, a clear dichotomy between patients with high and low cytotoxic lymphocyte abundance scores. Nearly all genes affecting survival found in the CL-Low patients had a positive impact, whereas nearly all genes affecting survival in the CL-High patients had a negative impact. This is not entirely unsuspected given we know that tumors attempt to evade anti-tumor immunity through various mechanisms [16]. Other groups have also demonstrated this in the context of MSI-H colorectal cancer, noting a significant upregulation of multiple negative regulators of immunity in these patients $[9,10]$. This data further underscores the need to develop and test new immune based therapy in patients with colorectal cancer tailored to patient specific factors. 


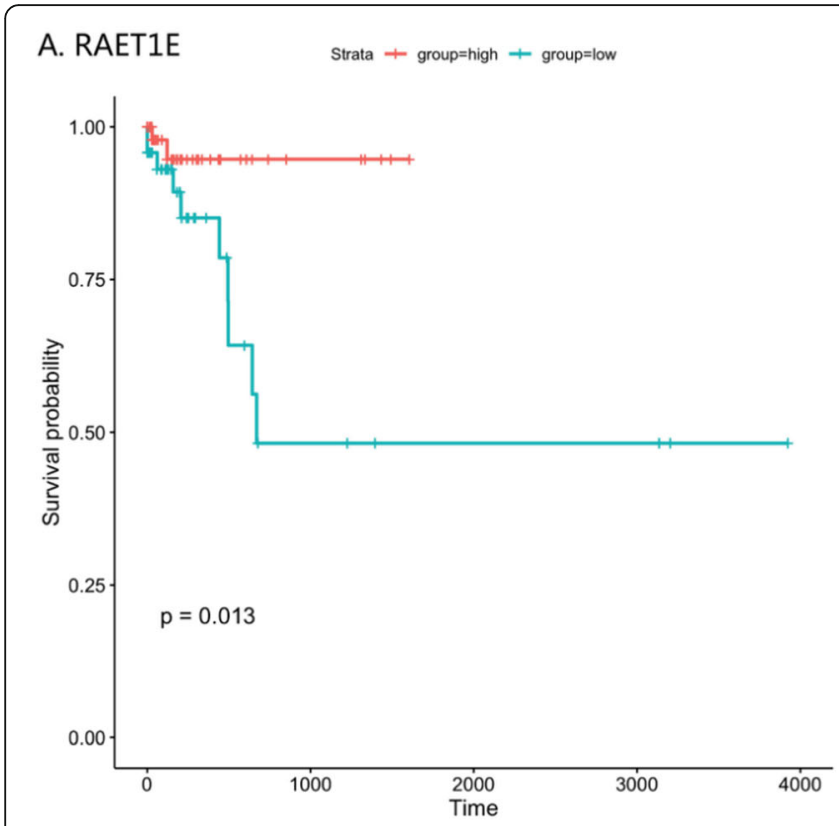

B. LAIR1(CD305) strata + group=high + group=low

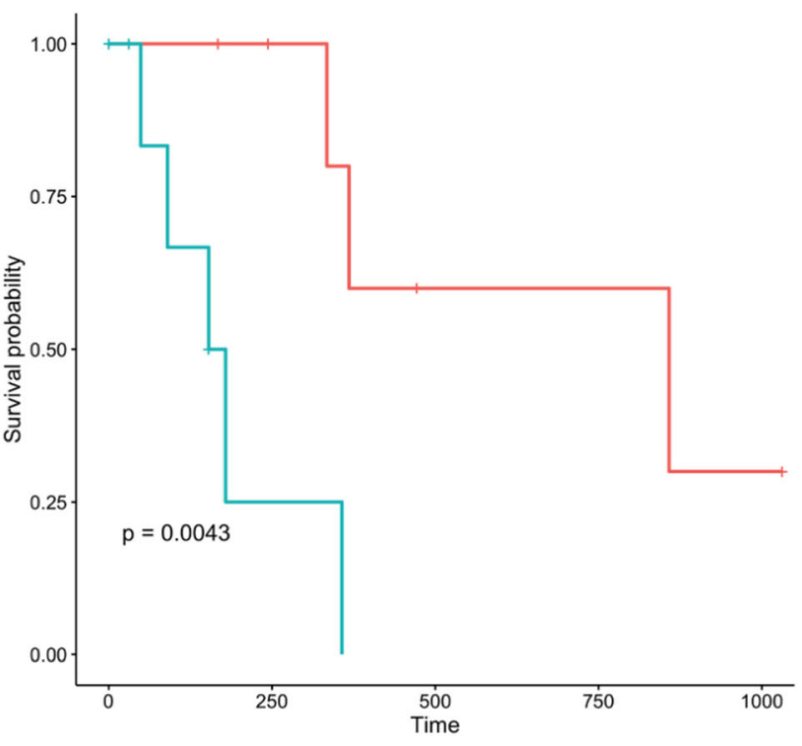

C. KLRC1

Strata + group=high + group=low

D. HCST

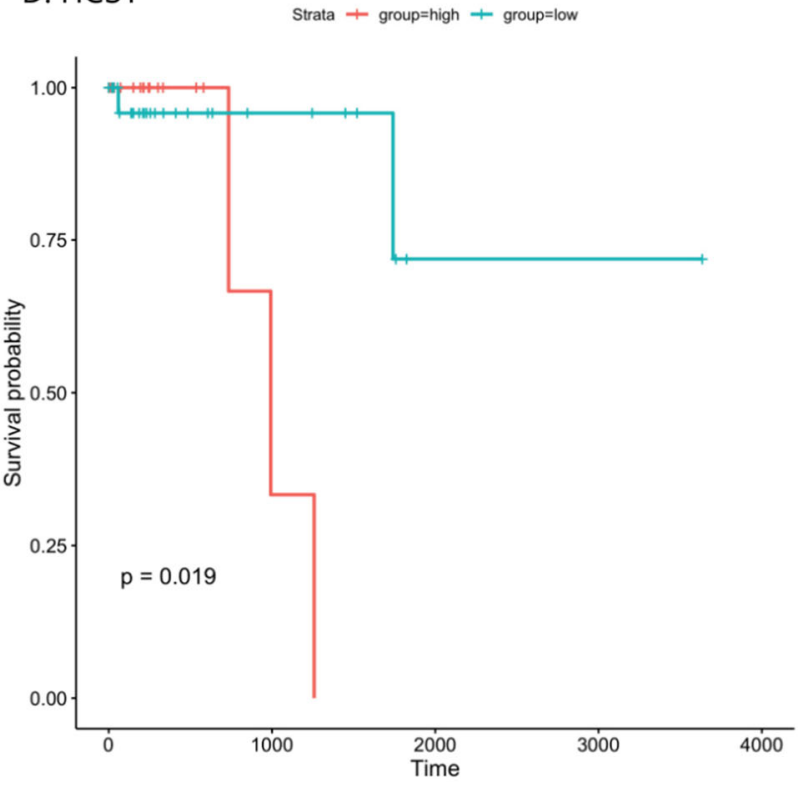

Fig. 3 Representative survival curves based on tumor location and stage. Survival curves with the $p$ values derived from Kaplan-Meier analysis. a RAET1E was positively associated with survival in right-sided colon cancer patients with high cytotoxic lymphocyte scores in early and localized stages; b LAIR1(CD305) was positively associated with survival in right-sided colon cancer patients with low cytotoxic lymphocyte scores in the metastatic stage; c KLRC1 was positively associated with survival in left-sided colon cancer patients with low cytotoxic lymphocyte scores in the early stage; d HCST was negatively associated with survival in rectal cancer patients with low cytotoxic lymphocyte scores in the localized group

In our survival analysis, we identified several potential targets for combination therapy. CD40L is a cell surface marker expressed on activated $\mathrm{T}$ cells that promotes maturation of antigen presenting cells, upregulating costimulatory molecules and activating antigen presentation machinery, and may represent the most attractive target identified in this work. In our analysis, this gene demonstrated a positive impact on survival in metastatic patients with low cytotoxic lymphocyte abundance scores. In preclinical models, CD40 agonists have demonstrated a significant ability to activate anti-cancer immunity, overcome immune checkpoint inhibition resistance, and work in concert with other immune based treatments [24]. Currently, a number of clinical trials are open studying these drugs in combination with other immune based treatments; however, none are specifically 
Table 2 Differentially expressed genes in the "Immunoregulatory interactions between a lymphoid and a non-lymphoid cell" pathway associated with survival grouped by pathologic stage, cytotoxic lymphocyte (CL) status, and anatomical location

Immunoregulatory interactions between a Lymphoid and a nonLymphoid cell

\begin{tabular}{|c|c|c|c|c|}
\hline Location & Stages & CLs Status & Gene Symbol & $P$-value \\
\hline \multirow[t]{22}{*}{ Right } & Early $(I, I)$ & High & RAET1E & 0.033 \\
\hline & & & LILRA1 & 0.031 \\
\hline & & & CD33 & 0.018 \\
\hline & & Low & N/A & \\
\hline & Localized $(I,|I| I \mid)$, & High & RAET1E & 0.013 \\
\hline & & & LILRAI & 0.0087 \\
\hline & & & LILRA4 & 0.042 \\
\hline & & & CD33 & 0.017 \\
\hline & & Low & $K L R C 1$ & 0.027 \\
\hline & Metastasis (IV) & High & MADCAM1 & 0.046 \\
\hline & & & $F C G R 2 B(C D 32)$ & 0.046 \\
\hline & & & SIGLEC8(CD329) & 0.046 \\
\hline & & & CLEC4G & 0.046 \\
\hline & & Low & CD96 & 0.027 \\
\hline & & & LILRB1 & 0.015 \\
\hline & & & ITGB2(CD18) & 0.033 \\
\hline & & & CD40LG & 0.029 \\
\hline & & & $\mathrm{C} 3$ & 0.018 \\
\hline & & & SIGLEC9 & 0.0071 \\
\hline & & & SH2D1A & 0.027 \\
\hline & & & LAIR1(CD305) & 0.0043 \\
\hline & & & CD300A & 0.0043 \\
\hline \multirow[t]{6}{*}{ Left } & Early & High & N/A & \\
\hline & & Low & KLRC1 & 0.025 \\
\hline & Localized & High & N/A & \\
\hline & & Low & N/A & \\
\hline & Metastasis & High & N/A & \\
\hline & & Low & CLEC2B & 0.046 \\
\hline \multirow[t]{8}{*}{ Rectum } & Early & High & N/A & \\
\hline & & Low & CLEC4G & 0.046 \\
\hline & Localized & High & N/A & \\
\hline & & Low & HCST & 0.019 \\
\hline & & & FCGR1A(CD64) & 0.039 \\
\hline & & & CLEC4G & 0.022 \\
\hline & Metastasis & High & N/A & \\
\hline & & Low & N/A & \\
\hline
\end{tabular}

CLs Cytotoxic lymphocytes; gene symbols with bold font indicate positive impact on patient's survival, gene symbols with italic font indicate negative impact on patient's survival directed at colorectal cancer patients. The fact that there are drugs available targeting this interaction may lend itself to rapid translation in these patients. Additionally, we found potentially attractive targets in CD96 and CD18 (ITGB2), each of which has demonstrated some significant impact on anti-tumor immunity in preclinical studies with the potential for translation in the future [25-27].

One limitation of this study is related to patient numbers and clinical data available, as with many database studies. Due to patient numbers, we included patients in Stage I and II in the analysis for both "early" and "local" disease. This was done to increase patient numbers assigned to each group and improve our analysis. Based on our results, we felt this helped to support findings in the "early" stage patients as the Stage III patients contributed $40-60 \%$ of "local" patients depending on disease location. Another important potential confounding factor, however, is significant heterogeneity in therapy, most notably in patients with metastatic disease (Stage IV). These were real world patients not treated on specific study protocols, so this heterogeneity in treatment may represent an impactful difference. Additionally, the patients included in this study did not receive immune based therapy, so the impact of cytotoxic lymphocyte infiltration on response to immune based treatments cannot be directly assessed. However, a number of studies have previously shown that cytotoxic lymphocyte infiltration in colorectal cancer predicts survival and response to therapy $[4,5,8,10]$, therefore augmenting anti-tumor immunity is likely to be impactful when considering combination with conventional treatments such as chemotherapy, or immune based therapy alone. Recent studies in cancer therapy have also begun to understand that the immune response is critical to the efficacy of chemotherapy and radiotherapy, further highlighting the need to understand altered immune pathways in cancer [28]. Despite these limitations, resources such as the TCGA, when combined with informatics-based analysis, yield highly impactful results that can be used to develop future human studies and inform translational pre-clinical studies. The goals of precision-based oncology will be best met by combining studies of all types to select both the best therapy for each patient, as well as the best patient for each therapy.

\section{Conclusion}

In this study, we integrate comprehensive RNA-seq data, clinical and pathologic data, and cytotoxic lymphocyte scores to determine pathways associated with immune response and survival in patients with colorectal cancer. We identified one pathway, "immunoregulatory interactions between a lymphoid and non-lymphoid cell", that was highly enriched and included in all tumor locations 
and stages. We then found specific genes associated with survival, primarily in patients with the worst survival, those with metastatic right-sided colon cancer, that may be targeted to improve therapy. Future studies will focus on further exploration of immune pathway interactions using multi-omics analysis in humans, and mechanistic studies of $\mathrm{T}$ lymphocyte recruitment and activation in murine models of colorectal cancer.

\section{Supplementary information}

Supplementary information accompanies this paper at https://doi.org/10. $1186 /$ s 12885-020-6513-4

Additional file 1: Figure S1. Comparison of cytotoxic lymphocyte scores in High (CL-high) and Low (CL-low) patients. ${ }^{* * *} p<0.0001$. Table S1. Distribution of cytotoxic lymphocyte abundance scores including median with $95 \% \mathrm{Cl}$ and quartiles for the entire patient cohort.

Additional file 2. This data file includes the results of all differentially expressed genes when comparing each tumor location and stage. This file also includes all results from the pathway enrichment analysis that are included in the visualization (Figure 2). Additionally, the survival analysis from all genes with a significant impact on survival is included in this file.

\section{Abbreviations}

CGP: Comprehensive genomic profiling; CL: Cytotoxic lymphocytes; CLHigh: Cytotoxic lymphocyte-high; CL-Low: Cytotoxic lymphocyte-low; COAD: Colon adenocarcinoma; CRC: Colorectal cancer; DEG: Differentially expressed genes; FDA: Food and Drug Administration; FDR: False discovery rate; ICl: Immune checkpoint inhibition; LSC: Left-sided colon cancer; MCP: Microenvironment cell populations; mRNA: Messenger ribonucleic acid; MS: Microsatellite stability; MSI-H: Microsatellite instability-high; MSIL: Microsatellite instability-low; MSS: Microsatellite stable; OS: Overall survival; READ: Rectal adenocarcinoma; REC: Rectal cancer; RNA-seq: Ribonucleic acid sequencing; RSC: Right-sided colon cancer; RSEM: RNA-Seq by Expectation Maximization; SD: Standard deviation; TCGA: The Cancer Genome Atlas; TMB: Tumor mutational burden

\section{Acknowledgements}

We would also like to acknowledge Dr. Timothy Hoffman, Dr. Emma Teixeiro, and Dr. Kevin Staveley-O'Carroll for mentorship and thoughtful discussion during the development, writing, and revision of this work.

\section{Authors' contributions}

All authors approve the submitted version of this manuscript. YS contributed to the design, acquisition and analysis, and interpretation of the data in this study. YS also contributed to the drafting and revision of the manuscript. YG contributed to the design and interpretation of the data in this study. YG also contributed to the revision of the manuscript. JJH contributed to the design and interpretation of the data in this study. CRS contributed to the design, acquisition and analysis, and interpretation of the data in this study CRS also contributed to the drafting and revision of the manuscript. JBM is the corresponding author of this study. JBM contributed to the design, acquisition and analysis, and interpretation of the data in this study. JBM also contributed to the drafting and revision of the manuscript.

\section{Funding}

This work was supported in part by Career Development Award \#IK2BX004346-01A1 (JBM) from the United States (US) Department of Veterans Affairs Biomedical Laboratory Research and Development Service and NIH KL2TR002346 ICTS Mentored Clinical Research Scholar Program (JBM). JJH is supported by the National Library of Medicine of the National Institutes of Health under award number 5T32LM012410. The content is solely the responsibility of the authors and does not necessarily represent the official views of the Department of Veterans' Affairs or the $\mathrm{NIH}$. The funding bodies had no role in study design, collection, analysis, interpretation of data or writing the manuscript.

\section{Availability of data and materials}

Full access to source data from the TCGA via FireCloud requires permission through dbgap. An application for full access to the TCGA data may be submitted here: https://dbgap.ncbi.nlm.nih.gov/aa/wga.cgi?page=login. All methods and $\mathrm{R}$ packages used in the preparation of this manuscript are available publicly (see below). For further information requests, please contact the corresponding author.

TCGA data:

https://firecloud.terra.bio/\#workspaces/broad-firecloud-tcga/TCGA_COAD_ ControlledAccess_V1-0_DATA

https://firecloud.terra.bio/\#workspaces/broad-firecloud-tcga/TCGA_READ_

ControlledAccess_V1-0_DATA

R package:

MCP-counter https://github.com/ebecht/MCPcounter

edgeR https://bioconductor.org/packages/release/bioc/html/edgeR.html clusterProfiler https://bioconductor.org/packages/release/bioc/html/ clusterProfiler.html

DOSE https://bioconductor.org/packages/release/bioc/html/DOSE.html ReactomePA https://bioconductor.org/packages/release/bioc/html/ ReactomePA.html

Survminer https://cran.r-project.org/web/packages/survminer/index.html

\section{Ethics approval and consent to participate}

In this study, we used the Cancer Genome Atlas, a publically available dataset, and therefore there was no new consent or approval required for this study.

\section{Consent for publication}

This study entirely uses publically available data used in multiple previous publications, so separate consent was not needed.

\section{Competing interests}

The authors declare that they have no competing interests.

\section{Author details}

${ }^{1}$ Harry S. Truman Memorial Veterans' Hospital, Columbia, MO, USA. ${ }^{2}$ Institute for Data Science and Informatics, University of Missouri, 238 Naka Hall, Columbia, MO 65211-2060, USA. ${ }^{3}$ Department of Surgery, University of Missouri School of Medicine, 1 Hospital Dr., Columbia, MO 65212, USA

Received: 22 August 2019 Accepted: 3 January 2020

Published online: 14 February 2020

\section{References}

1. Siegel RL, Miller KD, Jemal A. Cancer statistics, 2019. CA Cancer J Clin. 2019; 69(1):7-34.

2. Drake CG, Lipson EJ, Brahmer JR. Breathing new life into immunotherapy: review of melanoma, lung and kidney cancer. Nat Rev Clin Oncol. 2014; 11(1):24.

3. Le DT, Uram JN, Wang $H$, Bartlett BR, Kemberling $H$, Eyring AD, Skora AD, Luber BS, Azad NS, Laheru D, Biedrzycki B. PD-1 blockade in tumors with mismatch-repair deficiency. N Engl J Med. 2015;372(26):2509-20.

4. Becht E, Giraldo NA, Lacroix L, Buttard B, Elarouci N, Petitprez F, Selves J, Laurent-Puig $P$, Sautès-Fridman $C$, Fridman WH, De Reyniès A. Estimating the population abundance of tissue-infiltrating immune and stromal cell populations using gene expression. Genome Biol. 2016;17(1):218.

5. Mlecnik B, Bindea G, Angell HK, Maby P, Angelova M, Tougeron D, Church SE, Lafontaine L, Fischer M, Fredriksen T, Sasso M. Integrative analyses of colorectal cancer show immunoscore is a stronger predictor of patient survival than microsatellite instability. Immunity. 2016;44(3):698-711.

6. Wei SC, Duffy CR, Allison JP. Fundamental mechanisms of immune checkpoint blockade therapy. Cancer Discov. 2018:8(9):1069-86.

7. Le DT, Durham JN, Smith KN, Wang H, Bartlett BR, Aulakh LK, Lu S, Kemberling $\mathrm{H}$, Wilt $\mathrm{C}$, Luber BS, Wong F. Mismatch repair deficiency predicts response of solid tumors to PD-1 blockade. Science. 2017:357(6349):409-13.

8. Dudley JC, Lin MT, Le DT, Eshleman JR. Microsatellite instability as a biomarker for PD-1 blockade. Clin Cancer Res. 2016;22(4):813-20.

9. Grasso CS, Giannakis M, Wells DK, Hamada T, Mu XJ, Quist M, Nowak JA, Nishihara R, Qian ZR, Inamura K, Morikawa T. Genetic mechanisms of immune evasion in colorectal cancer. Cancer Discov. 2018;8(6):730-49. 
10. Llosa NJ, Cruise M, Tam A, Wicks EC, Hechenbleikner EM, Taube JM, Blosser RL, Fan $\mathrm{H}$, Wang $\mathrm{H}$, Luber BS, Zhang $\mathrm{M}$. The vigorous immune microenvironment of microsatellite instable colon cancer is balanced by multiple counter-inhibitory checkpoints. Cancer Discov. 2015;5(1):43-51.

11. Fabrizio DA, George TJ Jr, Dunne RF, Frampton G, Sun J, Gowen K, Kennedy M, Greenbowe J, Schrock AB, Hezel AF, Ross JS. Beyond microsatellite testing: assessment of tumor mutational burden identifies subsets of colorectal cancer who may respond to immune checkpoint inhibition. J Gastrointest Oncol. 2018;9(4):610.

12. Douillard JY, Siena S, Cassidy J, et al. Randomized, phase III trial of panitumumab with infusional fluorouracil, leucovorin, and oxaliplatin (FOLFOX4) versus FOLFOX4 alone as first-line treatment in patients with previously untreated metastatic colorectal cancer: the PRIME study. I Clin Oncol. 2010;28(31):4697-705.

13. Sinicrope FA, Mahoney MR, Smyrk TC, et al. Prognostic impact of deficient DNA mismatch repair in patients with stage III colon cancer from a randomized trial of FOLFOX-based adjuvant chemotherapy. J Clin Oncol. 2013;31(29):3664

14. Guinney J, Dienstmann R, Wang $X$, De Reyniès $A$, Schlicker $A$, Soneson $C$, Marisa L, Roepman P, Nyamundanda G, Angelino P, Bot BM. The consensus molecular subtypes of colorectal cancer. Nat Med. 2015;21(11):1350.

15. Loupakis F, Yang D, Yau L, Feng S, Cremolini C, Zhang W, Maus MK, Antoniotti C, Langer C, Scherer SJ, Müller T. Primary tumor location as a prognostic factor in metastatic colorectal cancer. J Natl Cancer Inst. 2015; 107(3). https://doi.org/10.1093/jnci/dju427.

16. Schreiber RD, Old LJ, Smyth MJ. Cancer immunoediting: integrating immunity's roles in cancer suppression and promotion. Science. 2011; 331(6024):1565-70.

17. Weinstein JN, Collisson EA, Mills GB, Shaw KR, Ozenberger BA, Ellrott $K$, Shmulevich I, Sander C, Stuart JM, Cancer Genome Atlas Research Network. The cancer genome atlas pan-cancer analysis project. Nat Genet. 2013; 45(10):1113.

18. Han J, Puri RK. Analysis of the cancer genome atlas (TCGA) database identifies an inverse relationship between interleukin-13 receptor a1 and a2 gene expression and poor prognosis and drug resistance in subjects with glioblastoma multiforme. J Neuro-Oncol. 2018;136(3):463-74.

19. Robinson MD, McCarthy DJ, Smyth GK. edgeR: a bioconductor package for differential expression analysis of digital gene expression data. Bioinformatics. 2010;26(1):139-40.

20. Fabregat A, Sidiropoulos K, Garapati P, et al. The reactome pathway knowledgebase. Nucleic Acids Res. 2015;44(D1):D481-7.

21. Kassambara A, Kosinski M, Biecek P. survminer: drawing survival curves using'ggplot2'. R package version 0.3; 2017. p. 1.

22. Therneau T. A package for survival analysis in $\mathrm{S}$. R package version 2.36-14; 2012

23. Petrelli F, Tomasello G, Borgonovo K, Ghidini M, Turati L, Dallera P, Passalacqua R, Sgroi G, Barni S. Prognostic survival associated with left-sided vs right-sided colon cancer: a systematic review and meta-analysis. JAMA Oncol. 2017;3(2):211-9.

24. Beatty GL, Li Y, Long KB. Cancer immunotherapy: activating innate and adaptive immunity through CD40 agonists. Expert Rev Anticancer Ther. 2017;17(2):175-86

25. Mittal D, Lepletier A, Madore J, Aguilera AR, Stannard K, Blake SJ, Whitehall VL, Liu C, Bettington ML, Takeda K, Long GV. CD96 is an immune checkpoint that regulates CD8+ T-cell antitumor function. Cancer Immunol Res. 2019;7(4):559-71.

26. Panni RZ, Herndon JM, Zuo C, Hegde S, Hogg GD, Knolhoff BL, Breden MA, Li X, Krisnawan VE, Khan SQ, Schwarz JK. Agonism of CD11b reprograms innate immunity to sensitize pancreatic cancer to immunotherapies. Sci Transl Med. 2019:11(499):eaau9240.

27. Iannello A, Thompson TW, Ardolino M, Marcus A, Raulet DH. Immunosurveillance and immunotherapy of tumors by innate immune cells. Curr Opin Immunol. 2016;38:52-8.

28. Galluzzi L, Buque A, Kepp O, Zitvogel L, Kroemer G. Immunological effects of conventional chemotherapy and targeted anticancer agents. Cancer Cell. 2015;28(6):690-714.

\section{Publisher's Note}

Springer Nature remains neutral with regard to jurisdictional claims in published maps and institutional affiliations.

\section{Ready to submit your research? Choose BMC and benefit from:}

- fast, convenient online submission

- thorough peer review by experienced researchers in your field

- rapid publication on acceptance

- support for research data, including large and complex data types

- gold Open Access which fosters wider collaboration and increased citations

- maximum visibility for your research: over $100 \mathrm{M}$ website views per year

At BMC, research is always in progress.

Learn more biomedcentral.com/submissions 